\title{
A Greedy Algorithm for the Unforecasted Energy Dispatch Problem with Storage in Smart Grids
}

\author{
Giorgos Georgiadis \\ Computer Science and Engineering \\ Chalmers University of Technology \\ Göteborg, Sweden \\ georgiog@chalmers.se
}

\author{
Marina Papatriantafilou \\ Computer Science and Engineering \\ Chalmers University of Technology \\ Göteborg, Sweden \\ ptrianta@chalmers.se
}

\begin{abstract}
Integration of renewable and distributed energy sources is today possible through the use of the Smart Grid but these technologies bring benefits as well as challenges, such as their intermittent nature that leads to utilization problems for the grid. On the other hand, upcoming storage technologies, such as electrical cars, hold the potential to store and utilize this intermittent supply at a later time but bring challenges of their own, for example efficient storage utilization and intermittent energy demand.

In this work we propose a novel modeling of the problem of unforecasted energy dispatch with storage as a scheduling problem of tasks on machines and an associated greedy algorithm with a guaranteed performance, along with an efficient algorithm for the problem. Finally, we outline an extensive simulation study for a variety of scenarios based on data from a large network of customers.
\end{abstract}

\section{Categories and Subject Descriptors}

F.2.2 [Nonnumerical Algorithms and Problems]: Scheduling algorithms

\section{Keywords}

online scheduling; resource allocation; smart grid

\section{INTRODUCTION}

In recent years there has been an organized effort on an international level to modernize the power grid by adding resilience properties, precise accounting and new services through the use of information technologies, collectively leading to a new type of grid commonly called smart grid. It is expected that these changes will enable the incorporation of renewable (e.g. photovoltaic arrays and wind generator farms) and distributed (e.g. electric car fleets) energy sources on a large scale but these technologies bring benefits as well as challenges.

While established models (cf [2] and bibliography therein) are able to accurately predict energy demand and compensate with sufficient supply, the intermittent nature of renewable energy sources, such as wind generator farms, challenges the way we utilize energy when it is available, compensate for when it is not and rely on weather forecasts for the grids' daily operation. On the other hand, distributed

Copyright is held by the author/owner(s).

e-Energy'13, May 21-24, 2013, Berkeley, California, USA.

ACM 978-1-4503-2052-8/13/05. energy sources such as electric car fleets can act as storage options and balance the demand and supply of electrical energy but bring challenges of their own, for example efficient storage utilization and intermittent energy demand. In the present work we focus on solutions that adapt the demand and use storage capabilities to mitigate the intermittent effects of renewable and distributed energy sources.

By drawing on the problem of scheduling tasks to machines, we model the problem of energy dispatch from producers to consumers in the distribution level of the grid, using an extended online load balancing problem. We present a novel scheduling and resource dispatching algorithm that is able to cope with the inherent unpredictability of renewable energy sources without the use of forecasts, as well as take advantage of available storage options in the grid. We also show analytically that the proposed algorithm is within a logarithmic factor of the optimal solution for the specific problem. Finally we conduct an extensive simulation study for a variety of scenarios based on data from a large number of customers and show that the presented algorithm is highly competitive to methods that use forecasts and assume total knowledge about the incoming load demand requests for the same problem.

\section{SYSTEM DEFINITION}

We focus on the distribution system of the grid as a high level abstraction, by considering a number of nodes being connected through a simple topology, for example low voltage power lines in a radial feeder configuration [3]. These nodes issue load demand requests on the feeder at irregular intervals, independently from each other, and energy is being dispatched from generation sites to satisfy the demand. For reasons of convention, we are assuming that requests are scheduled in hourly timeslots and are coming in a diurnal pattern, i.e. a demand refers to at most the next 24 hours. Note that we are considering both electrical and thermal services offered in two separate feeder lines on all nodes, an electrical and a thermal one respectively. In total, we identify three orthogonal axis to characterize load demand requests and we support demands of any of the eight types that these axis jointly define: elastic/inelastic, according to the ability to shift the demanded load over time or not ${ }^{1}$, electrical/thermal, depending on whether the demanded load can be serviced using only electrical energy or both

\footnotetext{
${ }^{1}$ Elastic loads can be scheduled to be serviced within a set of timeslots, while inelastic loads must be serviced necessarily in a specific time slot.
} 
electrical and thermal, and storage/simple, according to the ability to store the demanded load for future use or not.

The unforecasted energy dispatch problem with storage that is addressed here is the problem of dispatching generated electrical and thermal energy to end consumers in a way that minimizes peak energy consumption within a given time interval, without using forecasts and by taking into account any storage capabilities present.

\section{MODELING AND ALGORITHMIC AP- PROACH}

We model the unforecasted energy dispatch problem with storage to a problem of scheduling tasks to restricted machines $^{2}$ as follows. We regard load demand requests as an input sequence of tasks to be run on machines and the electrical and thermal feeder lines as the machines themselves. To capture the dimension of time, each feeder line corresponds to 24 machines, one for each hourly timeslot, leading to a total of 48 under this modeling. Each machine has the capability of storing energy if a storage task is run on it, and subsequent machines have a portion ${ }^{3}$ of this storage also available, to reflect the fact that stored energy is available in later timeslots. In total, the elastic/inelastic characteristic of demand requests is expressed in the set of machines that the demand is allowed to run, the electrical/thermal characteristic in the machines themselves and the storage/simple characteristic in the accumulated storage on the machines.

We consider the case that encapsulates the above modeling, where machines are defined as previously and can accumulate storage and tasks can either be simple (i.e. only inducing load on a machine) or storage (i.e. inducing load and generating storage). We call the resulting problem from this transformation an online load demand balancing problem with storage, which is the problem of assigning the tasks to the machines while minimizing the maximum load on the machines.

Based on this modeling, we propose the STORAGEGREEDY algorithm, which assigns each incoming task to the allowed machine that has the minimum load-storage difference (breaking ties arbitrarily). In practice, this is expressed on a system level and for a load demand request with specific restrictions by scheduling the request to be executed on the allowed timeslot with the minimum load-storage difference. Note here that the scheduling is taking place on the node that issued the request (where a copy of the algorithm is running), without a centralized decision center.

Following a methodology similar to [1], we show that the solution cost of our algorithm lies within a factor of $\lceil\log n\rceil+$ 1 when compared to the cost of the optimal algorithm, where $n$ is the number of machines (according to our modeling, $n=48)$. Note that no assumptions are made regarding the dissipation of the stored energy and the results apply both for dissipating and non-dissipating storage.

\section{EXPERIMENTAL STUDY}

Here we outline a small part of the study that was conducted using the reported Swedish load demand mix for

${ }^{2}$ I.e. where a task is allowed to run only on selected machines.

${ }^{3}$ Depending on whether losses in storage are being considered. households, using data collected from 400 households over the course of approximately 4 years, on behalf of the Swedish Energy Agency [4]. These data were extrapolated in order to model different households (customer profiles) and different types of loads (i.e. elastic/inelastic, storage etc). A number of scenarios were subsequently created, with a varying amount of houses and penetration of renewables and flexible loads, ranging from a mix of $65 \%-27 \%-8 \%$ inelastic electric, inelastic thermal and elastic electric loads respectively (business-as-usual scenario) to a mix of $45 \%-12 \%-12 \%-8 \%$ $3 \%$ inelastic electric, inelastic thermal, elastic electric, elastic thermal loads and elastic thermal, elastic electric storage respectively (smart house/neighborhood scenario).

During our experiments we aimed at minimizing the peak consumption and focused on the comparison between our STORAGEGREEDY algorithm and a simple, yet powerful algorithm for the same problem that uses forecasts, called the LPT algorithm: tasks are sorted globally by decreasing processing time and each task is assigned to the machine that has the least load (breaking ties arbitrarily) (see figure 1).
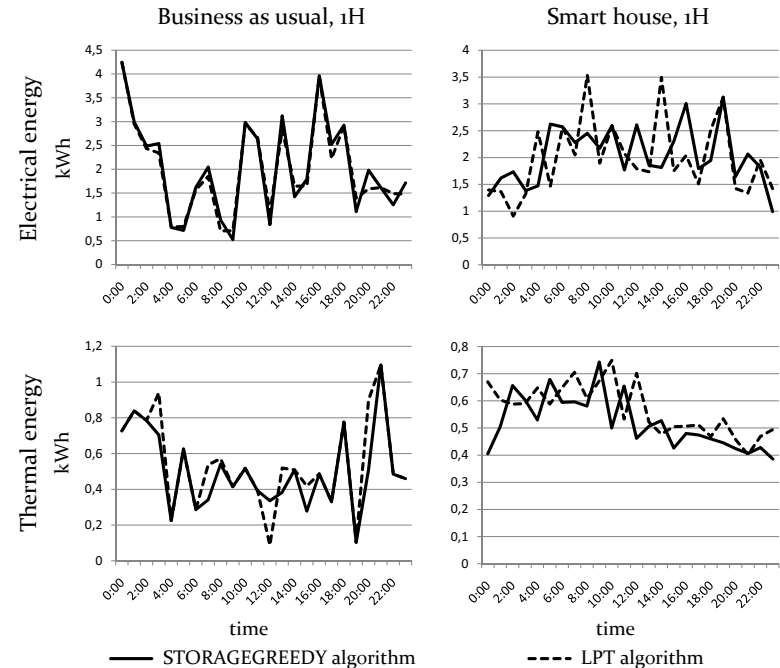

Figure 1: Comparison of load demand curves for the Business as usual and Smart house scenarios for one household

It is easy to see that for the business as usual scenario the two algorithms have comparable performance. However, under the smart house scenario the STORAGEGREEDY algorithm succeeds in lowering peak consumption by taking advantage of storage, even though the LPT algorithm has full access to forecasted demands.

\section{REFERENCES}

[1] A. Borodin and R. El-Yaniv. Online Computation and Competitive Analysis. Cambridge University Press, Apr. 1998.

[2] W. H. Kersting. Distribution System Modeling and Analysis. CRC Press, 1 edition, Aug. 2001.

[3] A. von Meier. Electric Power Systems: A Conceptual Introduction. Wiley-IEEE Press, 1 edition, July 2006.

[4] J. P. Zimmermann. End-use metering campaign in 400 households in sweden assessment of the potential electricity savings. Energimyndigheten, Sweden, 2009. 